\title{
Cyclophosphamide mechanism of action in preclinical tecemotide studies
}

\author{
Ken Hance ${ }^{1 *}$, Robert Tighe ${ }^{1}$, Jin Qi ${ }^{1}$, William Hastings ${ }^{1}$, Guozhong Qin ${ }^{1}$, Bo Marelli ${ }^{1}$, Hong Wang ${ }^{1}$, Huakui Yu ${ }^{1}$, \\ Yanping Zhang ${ }^{1}$, Xiaomei Xu', Wilson Guzman', Masie Wong ${ }^{1}$, Giorgio Kradjian', Beatrice Brunkhorst', \\ Helen Sabzevari ${ }^{1}$, Yan Lan ${ }^{1}$, Robert Hofmeister ${ }^{1}$, Michael Wolf ${ }^{2}$ \\ From Society for Immunotherapy of Cancer 28th Annual Meeting \\ National Harbor, MD, USA. 8-10 November 2013
}

\section{Background}

Tecemotide is a MUC1 antigen-specific therapeutic cancer vaccine. In phase III clinical studies, delivery of tecemotide is preceded by a single low dose of cyclophosphamide (CPA) to inhibit regulatory $\mathrm{T}$ cells (Tregs) and enhance the response to the tumor-associated antigen. Here, we investigated effects of CPA on the immune environment and tumor growth in preclinical murine models.

\section{Methods}

The effect of CPA was investigated in tumor-free human MUC1 transgenic mice and anti-tumor responses were evaluated in the mice engrafted with syngeneic colorectal and ovarian cancer cells expressing human MUC1. The mice received either vehicle control, CPA $(100 \mathrm{mg} /$ $\mathrm{kg})$, tecemotide $(100 \mu \mathrm{g})$ or CPA + tecemotide. The immune cell phenotype and function in the spleen were assessed on Days 1, 3 and 7 post-administration.

\section{Results}

The single administration of CPA led to a reduction of the absolute numbers of splenocytes, including $\mathrm{CD} 8^{+}$cells and Tregs ( $\mathrm{p}<0.05$ on Days 1, 3 and 7 vs. control). But while by Day 7 post-administration the total number of splenocytes and $\mathrm{CD} 8^{+} \mathrm{T}$ cells partially recovered, the number of Tregs stayed low. This resulted in a higher $\mathrm{CD} 8^{+} /$Treg ratio. Further, Tregs exhibited significantly reduced suppressor activity on a per cell basis as observed on Day 3. In the MC38/MUC1 colorectal cancer model, CPA alone reduced the growth of tumors, which was enhanced by the combination of CPA + tecemotide (2-way ANOVA,

${ }^{1}$ ImmunoOncology, EMD Serono Research \& Development Institute Inc., Billerica, MA, USA

Full list of author information is available at the end of the article $\mathrm{p}<0.05)$. Tecemotide monotherapy and vehicle control did not show an effect. Likewise, mice treated with the combination survived longer (median survival $31 \mathrm{~d}$ vs. $19 \mathrm{~d}$ with tecemotide; $24.5 \mathrm{~d}$, CPA; $19.5 \mathrm{~d}$, control; $\mathrm{P}_{\mathrm{TREND}}=0.04$ ). The combination therapy also significantly increased the precursor frequency of endogenous P15E-antigen-specific $\mathrm{CD}^{+} \mathrm{T}$ cells and IFN- $\gamma$ production indicating antigen spreading. In the $\mathrm{MUC1}^{+}$orthotopic ovarian cancer model MOSEC/MUC1, tecemotide inhibited tumor growth (2-way ANOVA, $\mathrm{p}<0.05$ ) and the development of abdominal ascites. Tecemotide significantly elevated BP25specific $\mathrm{CD} 4^{+} \mathrm{T}$ cell IFN- $\gamma$ production as compared to the control or CPA, a response that was significantly enhanced by the combination of tecemotide with CPA.

\section{Conclusions}

Besides its direct cytotoxic effects on tumor cells, CPA can reduce immunosuppressive mechanisms in the tumor and thereby create a favorable immune environment for the combination with tecemotide to exert its activity. These findings provide mechanistic evidence to support the use of low-dose CPA as a preconditioning agent designed to potentiate the immune and antitumor efficacy of tecemotide.

\section{Authors' details \\ ${ }^{1}$ ImmunoOncology, EMD Serono Research \& Development Institute Inc., Billerica, MA, USA. ${ }^{2}$ Merck KGaA, Darmstadt, Germany.}

Published: 7 November 2013

doi:10.1186/2051-1426-1-S1-P75

Cite this article as: Hance et al:: Cyclophosphamide mechanism of action in preclinical tecemotide studies. Journal for ImmunoTherapy of Cancer 2013 1(Suppl 1):P75. 